\title{
Echogenic foci in thyroid nodules: diagnostic performance with combination of TIRADS and echogenic foci
}

\author{
Su Min Ha ${ }^{1,4}$, Yun Jae Chung ${ }^{2^{*}}$, Hye Shin Ahn ${ }^{1}$, Jung Hwan Baek ${ }^{3}$ and Sung Bin Park
}

\begin{abstract}
Background: The malignancy risks of various echogenic foci in thyroid nodules are not consistent. The association between malignancy and echogenic foci and various Thyroid Imaging Reporting and Data System (TIRADS) in thyroid nodules has not been evaluated. We evaluated the malignancy probability and diagnostic performance of thyroid nodules with various echogenic foci and in combination with TIRADS.

Methods: This retrospective study was approved by Institutional Review Board. The data were retrospectively collected from January 2013 to December 2014. In total, 954 patients (mean age, 50.8 years; range, 13-86 years) with 1112 nodules were included. Using $x^{2}$ test, we determined the prevalence of benign and malignant nodules among those with and without echogenic foci; we associated each of 6 echogenic foci types with benign and malignant nodules. Diagnostic performance was compared between the 6 types alone and in combination with various TIRADS.
\end{abstract}

Results: Among 1112 nodules, 390 nodules (35.1\%) were found to have echogenic foci, and 722 nodules (64.9\%) were not. Among nodules with echogenic foci, 254 nodules (65.1\%) were malignant. The punctate echogenic foci with comet-tail artifact showed malignancy rate of $77.8 \%$ in solid and predominantly solid nodules. Our study demonstrated relatively low PPV (33.3-56.4\%) in nodules with large echogenic foci without shadowing, macrocalcification, and peripheral curvilinear or eggshell echogenic foci with or without shadowing. However, when combined with high suspicion category of TIRADS, PPV increased to 50.0-90.9\%.

Conclusion: Combination with TIRADS with different types of echogenic foci offer better stratification of the malignancy risk.

Keywords: Thyroid nodules, Ultrasound, Thyroid cancer, Thyroid TIRADS, Echogenic foci

\section{Background}

Although widespread use of ultrasonography (US) has exponentially increased thyroid nodule detection to about $19.0-67.0 \%$, malignancy is found in only about 9.0-15.0\% of nodules evaluated using fine-needle aspiration (FNA) [1-3]. To minimize potential harm from overuse of FNA, the Thyroid Imaging Reporting and Data System (TIRADS) was developed for thyroid nodule risk stratification [4-6]. One such model was recently published by the Korean Society of Thyroid

\footnotetext{
* Correspondence: yjchung@cau.ac.kr

2Department of Internal Medicine, Chung-Ang University Hospital,

Chung-Ang University College of Medicine, 102, Heukseok-ro, Dongjak-gu,

Seoul 06973, Republic of Korea

Full list of author information is available at the end of the article
}

Radiology (KSThR) named the Korean TIRADS [7], and was validated prospectively in a multi-center study [8]. The American Thyroid Association (ATA) management guidelines for thyroid nodules also stratified the risk of malignancy into five categories [5]. Meanwhile, Choi et al. [9] developed a web-based automatic scoring risk stratification system using US characteristics. This system also classifies nodules according to various guidelines, such as the French TIRADS [10], ATA guidelines [5], and Korean TIRADS [7]. The various TIRADS include "echogenic foci" as a criterion for differentiating benign and malignant thyroid nodules. Echogenic foci within the thyroid nodule are common on US with $14.0-55.0 \%$ incidence [11-14] and 29.0-59.0\% 
malignancy rate $[11,13-16]$. Indeed, there are various patterns of echogenic foci [12, 15, 17], but are often poorly defined and simply referred to as calcifications. The clinical significance of other types of echogenic foci is not yet clear, except for punctate echogenic foci what many authors call as microcalcifications [18-21]. Microcalcifications are generally accepted as the most reliable indicator of malignancy because they mostly represent psammoma bodies [12].

The recently published American College of Radiology (ACR) TIRADS defined echogenic foci into five categories: punctate foci with no posterior artifact, echogenic foci with small comet-tail artifact $(\leq 1 \mathrm{~mm}$ long), echogenic foci with large comet-tail artifact (> $1 \mathrm{~mm}$ long), peripheral echogenic foci with acoustic shadowing, and clumplike echogenic foci with acoustic shadowing [20]. They insisted that echogenic foci with small comet-tail artifact in solid hypoechoic nodule should be distinguished from the large comet-tail artifacts in the cystic components of a thyroid nodule [22]. And large comet-tail artifacts in hypoechoic nodules to be viewed with suspicion. According to Korean-TIRADS developed by KSThR, the malignancy risk of microcalcification is high in the solid hypoechoic nodules, but intermediate risk in the partially cystic and iso- and hyperechoic nodules .

The malignancy risks of various echogenic foci in thyroid nodules are not consistent; evidence is limited with variable value. Furthermore, the association between malignancy and echogenic foci and various TIRADS in thyroid nodules has not been evaluated. Our study evaluated the malignancy probability and diagnostic performance in differentiating benign and malignant nodules with various types of echogenic foci and when combined with various TIRADS.

\section{Methods}

This retrospective study was approved by the Institutional Review Board; informed consent was waived for data evaluation. Written informed consent for routine thyroid US and US-guided procedures was obtained from all patients before each US examination.

\section{Study population}

The patient cohort was retrospectively collected in patients undergoing US from January 2013 to December 2014. A total of 2703 consecutive nodules $(\geq 5 \mathrm{~mm}$ ) were selected for a database of thyroid nodules that underwent US-guided core-needle biopsy (CNB) or FNA. Among them, 1591 nodules with indeterminate or non-diagnostic results and without follow-up for final diagnosis were excluded. Finally, a total of 954 patients (mean age, 50.8 years; range, 13-86 years) with 1112 nodules $(\geq 5 \mathrm{~mm}$ ) were included in our study. For each
Table 1 Rate of Malignancy by Thyroid Nodule Echogenic Foci Type

\begin{tabular}{lllll}
\hline & Total $(n=390)$ & Benign $(n=136)$ & Malignant $(n=254)$ & $P$ value \\
\hline Type 1 & $207(53.1)$ & $54(26.1)$ & $153(73.9)$ & $<0.001$ \\
Type 2 & $54(13.8)$ & $12(22.2)$ & $42(77.8)$ & 0.452 \\
Type 3 & $94(24.1)$ & $41(43.6)$ & $53(56.4)$ & 0.041 \\
Type 4 & $27(6.9)$ & $16(59.3)$ & $11(40.7)$ & 0.006 \\
Type 5 & $21(5.4)$ & $14(66.7)$ & $7(33.3)$ & 0.002 \\
Type 6 & $41(10.5)$ & $11(26.8)$ & $30(73.2)$ & 0.253 \\
\hline
\end{tabular}

Data indicate the number of lesions

Numbers in parentheses indicate percentages

Type $1=$ Punctate echogenic foci $(\leq 1 \mathrm{~mm})$ with or without posterior shadowing

Type 2 = Punctate echogenic foci with comet-tail artifact

Type 3 = Large echogenic foci $(>1 \mathrm{~mm}$ ) without shadowing

Type $4=$ Macrocalcification (defined as large echogenic foci $(>1 \mathrm{~mm}$ ) with shadowing)

Type $5=$ Peripheral curvilinear or eggshell echogenic foci with or without shadowing

Type $6=$ Nodules with more than one type of echogenic foci

thyroid nodule, the final diagnosis was determined either by histopathology or radiological follow-up. For malignant nodules $(n=417)$, the pathological diagnosis was confirmed by surgery $(n=413)$ or CNB $(n=4)$ [23]. For benign nodules $(n=695)$, the pathological diagnosis was confirmed by surgery $(n=65)$, repeated FNA or CNB at least twice with benign results $(n=41)$, or a benign result on FNA or CNB with no change or reduced size seen on follow-up US more than $>12$ months later $(n=$ 589). The interpretation of FNA was based on the Bethesda system for reporting thyroid cytopathology [24], and the 6 categories of a CNB pathology reporting system were used for interpretation of CNB [25].

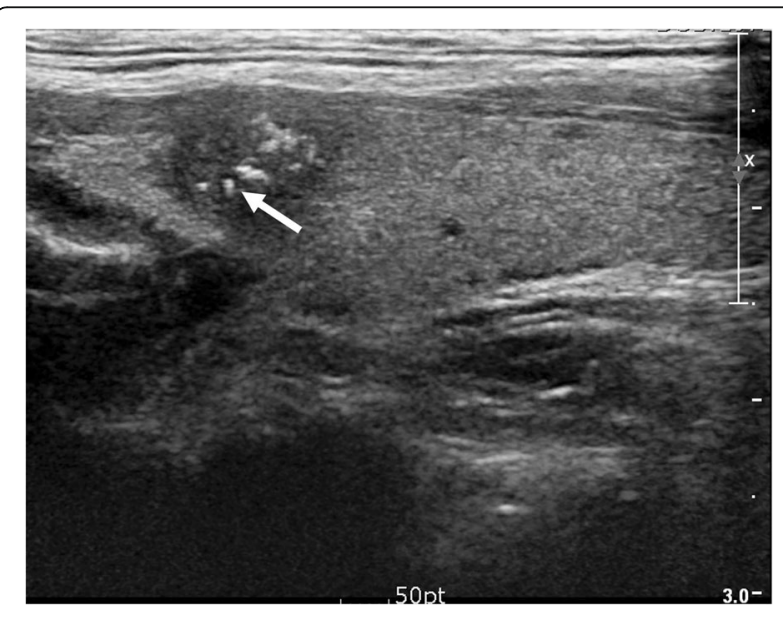

Fig. 1 Echogenic foci associated with malignancy. 41-year-old male with thyroid nodule. Ultrasound image shows a $1.0 \mathrm{~cm}$ hypoechoic, solid nodule with spiculated margin. Multiple echogenic foci (Type 6) are present, including echogenic foci with comet-tail artifact (Type 2, arrow). Biopsy result was papillary carcinoma and was confirmed at surgery 
Table 2 Comparison of Malignancy Rate According to Various Guidelines

\begin{tabular}{lllll}
\hline & TIRADS & Suspicious nodule on US & Confirmed as cancer & Malignancy rate \\
\hline Total $(n=390)$ & Web-based TIRADS $(\geq 8)$ & $199(51.0)$ & $178(70.1)$ & $70.1 \%$ \\
& K-TIRADS High Suspicion & $226(57.9)$ & $198(78.0)$ & $78.0 \%$ \\
& ATA guidelines High Suspicion & $235(60.3)$ & $205(96.2)$ & $96.2 \%$ \\
& Russ et al. $(\geq 4$ B) & $278(71.3)$ & $217(85.4)$ & $85.4 \%$ \\
\hline
\end{tabular}

Data indicate the number of lesions

Numbers in parentheses indicate percentages

TIRADS thyroid image reporting and data system, ATA american thyroid association

\section{Ultrasound examination}

Ultrasound images for the evaluation of thyroid nodules were obtained using an iU22 ultrasound system (Philips Healthcare, Bothell, WA) equipped with a 50-mm linear array transducer with a bandwidth of 7-12 MHz. The scanning protocol in all cases included both transverse and longitudinal real-time imaging of thyroid nodules. US images were retrospectively reviewed by two radiologists (S.M.H., H.S.A) who had 8-10 years of experience in performing thyroid US. Images were reviewed with no previous knowledge of the biopsy result or final diagnosis and assessed the US features of thyroid nodules-internal content, echogenicity, margin, shape, presence or absence echogenic foci and comet-tail artifact. We excluded vascularity as a criterion.

The internal content of a nodule was categorized as solid (pure solid or nearly entirely solid), predominantly solid ( $<50 \%$ of the cystic portion), predominantly cystic (> 50\% of the cystic portion), or cystic (no obvious solid content). Echogenicity of the solid portion was classified as hyper- or iso-echogenicity, hypoechogenicity, or marked hypoechogenicity. When the echogenicity of the nodule was similar to that of the surrounding thyroid parenchyma, it was classified as isoechogenicity. Hypoechogenicity was defined as decreased echogenicity compared to the thyroid parenchyma. Marked hypoechogenicity was defined as decreased echogenicity compared to that of the strap muscles. The nodule shape was categorized as follows: ovoid to round (when the anteroposterior diameter of the nodule was equal to or less than its transverse diameter on a transverse or longitudinal plane); taller than wide (when the anteroposterior diameter of a nodule was longer than its transverse diameter on a transverse or longitudinal plane); or irregular (when a nodule was neither ovoid to round nor taller than wide). Margins were classified as well-defined smooth, microlobulated or spiculated, or ill-defined.

Nodules that did not have echogenic foci were kept for overall comparison. The nodules with echogenic foci were classified into the following 6 types: Type 1.Punctate echogenic foci $(\leq 1 \mathrm{~mm})$ with or without posterior shadowing, Type 2. Punctate echogenic foci with comet-tail artifact, Type 3. Large echogenic foci (>1

Table 3 Diagnostic Performance of US Characteristics for the Prediction of Malignancy in Thyroid Nodules with Echogenic Foci alone and TIRADS alone

\begin{tabular}{|c|c|c|c|c|c|c|c|c|c|}
\hline & & \multirow[t]{2}{*}{ Total } & \multirow[t]{2}{*}{ Benign } & \multirow[t]{2}{*}{ Malignant } & \multicolumn{5}{|c|}{ Diagnostic Performance } \\
\hline & & & & & Sensitivity & Specificity & Accuracy & PPV & NPV \\
\hline \multirow[t]{6}{*}{ Echogenic Foci } & Type 1 & 207 & 54 & 153 & 60.2 & 60.3 & 60.3 & 73.9 & 44.8 \\
\hline & Type 2 & 54 & 12 & 42 & 27.5 & 77.8 & 40.6 & 77.8 & 27.5 \\
\hline & Type 3 & 94 & 41 & 53 & 20.9 & 69.9 & 37.9 & 56.4 & 32.1 \\
\hline & Type 4 & 27 & 16 & 11 & 4.3 & 88.2 & 33.6 & 40.7 & 33.1 \\
\hline & Type 5 & 21 & 14 & 7 & 2.8 & 89.7 & 33.1 & 33.3 & 33.1 \\
\hline & Type 6 & 41 & 11 & 30 & 11.8 & 91.9 & 39.7 & 73.2 & 35.8 \\
\hline \multirow[t]{4}{*}{ TIRADS } & Web-based TIRADS( $\geq 8$ ) & 199 & 21 & 178 & 70.1 & 84.6 & 75.1 & 89.4 & 60.2 \\
\hline & K-TIRADS High Suspicion & 226 & 28 & 198 & 78.0 & 79.4 & 78.5 & 87.6 & 65.9 \\
\hline & ATA guidelines High Suspicion & 235 & 30 & 205 & 96.2 & 56.5 & 86.5 & 87.2 & 83.0 \\
\hline & Russ et al. ( $\geq 4 \mathrm{~B})$ & 278 & 61 & 217 & 85.4 & 55.1 & 74.9 & 78.1 & 67.0 \\
\hline
\end{tabular}

TIRADS thyroid image reporting and data system, ATA american thyroid association, PPV positive predictive value, NPV negative predictive value Type 1 = Punctate echogenic foci $(\leq 1 \mathrm{~mm})$ with or without posterior shadowing

Type 2 = Punctate echogenic foci with comet-tail artifact

Type 3 = Large echogenic foci $(>1 \mathrm{~mm}$ ) without shadowing

Type 4 = Macrocalcification (defined as large echogenic foci (>1 mm) with shadowing)

Type $5=$ Peripheral curvilinear or eggshell echogenic foci with or without shadowing

Type $6=$ Nodules with more than one type of echogenic foci 
Table 4 Diagnostic Performance of US Characteristics for the Prediction of Malignancy in Thyroid Nodules with Echogenic foci Types with TIRADS Combination (High suspicion)

\begin{tabular}{|c|c|c|c|c|c|c|c|c|c|}
\hline \multirow{2}{*}{$\begin{array}{l}\text { Echogenic } \\
\text { foci }\end{array}$} & \multirow[t]{2}{*}{ TIRADS } & \multirow[t]{2}{*}{ Total } & \multirow[t]{2}{*}{ Benign } & \multirow[t]{2}{*}{ Malignant } & \multicolumn{5}{|c|}{ Diagnostic Performance } \\
\hline & & & & & Sensitivity & Specificity & Accuracy & PPV & NPV \\
\hline \multirow[t]{4}{*}{ Type 1} & + Web-based TIRADS $(\geq 8)$ & 148 & 14 & 134 & 52.8 & 89.7 & 65.6 & 90.5 & 50.4 \\
\hline & + K-TIRADS High Suspicion & 156 & 19 & 137 & 53.9 & 86.0 & 65.1 & 87.8 & 50.0 \\
\hline & + ATA High Suspicion & 163 & 21 & 142 & 66.7 & 69.6 & 67.4 & 87.1 & 40.3 \\
\hline & + Russ et al. ( $\geq 4 \mathrm{~B})$ & 189 & 41 & 148 & 58.3 & 69.9 & 62.3 & 78.3 & 47.3 \\
\hline \multirow[t]{4}{*}{ Type 2} & + Web-based TIRADS $(\geq 8)$ & 37 & 3 & 34 & 22.2 & 94.4 & 41.1 & 91.9 & 30.0 \\
\hline & +K-TIRADS High Suspicion & 40 & 6 & 34 & 22.2 & 88.9 & 39.6 & 85.0 & 28.7 \\
\hline & + ATA High Suspicion & 46 & 7 & 39 & 27.1 & 75.9 & 35.3 & 84.8 & 17.3 \\
\hline & + Russ et al. ( $\geq 4 \mathrm{~B})$ & 47 & 8 & 39 & 25.5 & 85.2 & 41.1 & 83.0 & 28.8 \\
\hline \multirow[t]{4}{*}{ Type 3} & + Web-based TIRADS $(\geq 8)$ & 22 & 2 & 20 & 7.9 & 98.5 & 39.5 & 90.9 & 36.4 \\
\hline & +K-TIRADS High Suspicion & 37 & 6 & 31 & 12.2 & 95.6 & 41.3 & 83.8 & 36.8 \\
\hline & + ATA High Suspicion & 38 & 6 & 32 & 15.0 & 91.3 & 33.7 & 84.2 & 25.8 \\
\hline & + Russ et al. ( $\geq 4 \mathrm{~B})$ & 49 & 13 & 36 & 14.2 & 90.4 & 40.8 & 73.5 & 36.1 \\
\hline \multirow[t]{4}{*}{ Type 4} & + Web-based TIRADS $(\geq 8)$ & 2 & 1 & 1 & 0.4 & 99.3 & 34.9 & 50.0 & 34.8 \\
\hline & +K-TIRADS High Suspicion & 4 & 1 & 3 & 1.2 & 99.3 & 35.4 & 75.0 & 35.0 \\
\hline & + ATA High Suspicion & 4 & 1 & 3 & 1.4 & 98.6 & 25.2 & 75.0 & 24.5 \\
\hline & + Russ et al. ( $\geq 4 \mathrm{~B})$ & 6 & 2 & 4 & 1.6 & 98.5 & 35.4 & 66.7 & 34.9 \\
\hline \multirow[t]{4}{*}{ Type 5} & + Web-based TIRADS $(\geq 8)$ & 6 & 2 & 4 & 1.6 & 98.5 & 35.4 & 66.7 & 34.9 \\
\hline & +K-TIRADS High Suspicion & 5 & 1 & 4 & 1.6 & 99.3 & 35.6 & 80.0 & 35.1 \\
\hline & + ATA High Suspicion & 5 & 1 & 4 & 1.9 & 98.6 & 25.5 & 80.0 & 24.5 \\
\hline & + Russ et al. ( $\geq 4 \mathrm{~B})$ & 6 & 2 & 4 & 1.6 & 98.5 & 35.4 & 66.7 & 34.9 \\
\hline \multirow[t]{4}{*}{ Type 6} & + Web-based TIRADS $(\geq 8)$ & 21 & 2 & 19 & 7.5 & 98.5 & 39.2 & 90.5 & 36.3 \\
\hline & +K-TIRADS High Suspicion & 24 & 1 & 23 & 9.1 & 99.3 & 40.5 & 95.8 & 36.9 \\
\hline & + ATA High Suspicion & 25 & 1 & 24 & 11.3 & 98.6 & 32.6 & 96.0 & 26.5 \\
\hline & + Russ et al. ( $\geq 4 \mathrm{~B})$ & 28 & 3 & 25 & 9.8 & 97.8 & 40.5 & 89.3 & 36.7 \\
\hline
\end{tabular}

TIRADS thyroid image reporting and data system, ATA american thyroid association, $P P V$ positive predictive value, NPV negative predictive value Type $1=$ Punctate echogenic foci $(\leq 1 \mathrm{~mm})$ with or without posterior shadowing Type 2 = Punctate echogenic foci with comet-tail artifact

Type 3 = Large echogenic foci $(>1 \mathrm{~mm})$ without shadowing

Type 4 = Macrocalcification (defined as large echogenic foci $(>1 \mathrm{~mm}$ ) with shadowing)

Type $5=$ Peripheral curvilinear or eggshell echogenic foci with or without shadowing

Type $6=$ Nodules with more than one type of echogenic foci

$\mathrm{mm}$ ) without shadowing, Type 4. Macrocalcification (defined as large echogenic foci ( $>1 \mathrm{~mm}$ ) with shadowing), Type 5. Peripheral curvilinear or eggshell echogenic foci with or without shadowing, and Type 6. More than one type of echogenic foci. We calculated the probability of malignancy using various malignant risk systems, such as web-based TIRADS, K-TIRADS, ATA guidelines [5], and TIRADS of Russ et al. [26]. Suspicious nodules were defined as those with a score of $\geq 8$ with the web-based TIRADS, high suspicion with K-TIRADS, high suspicion with the ATA guidelines [5], and a score of $\geq 4 \mathrm{~B}$ with the TIRADS proposed by Russ et al. [26].

\section{Data and statistical analysis}

Multivariate logistic regression analysis was used to estimate the malignancy risk associated with US findings in thyroid nodules with and without echogenic foci. Using the $x^{2}$ test, we determined the overall prevalence of benign and malignant nodules among those with 6 echogenic foci types and by various guidelines. Diagnostic performance was evaluated by echogenic foci types alone, and in combination with various TIRADS. We calculated the sensitivity, specificity, negative predictive value (NPV), positive predictive value (PPV) and accuracy. The PPV between two classifications (1. nodules with echogenic foci and high suspicion TIRADS category, 2. nodules with echogenic foci and non-high suspicion TIRADS category) was compared using $x^{2}$ or Fisher's exact test. All statistical analyses were carried out using SPSS version 23.0 (IBM Corp., Armonk, NY, USA). Two-tailed $p$ values $<0.05$ were considered to be statistically significant. 


\section{Result}

Among the 1112 nodules, 390 (35.1\% [390 of 1112]) were found to have echogenic foci on US, and 722 (64.9\% [722 of 1112]) were not. Among 390 nodules with echogenic foci, 254 (65.1\% [254 of 390]) were malignant, and 136 (34.9\% [136 of 390]) were benign. Among 722 nodules without echogenic foci, 163 (22.6\% [163 of 722]) were malignant, and 559 (77.4\% [559 of 722]) were benign. There was a significant statistical difference $(22.6 \%$ vs $65.1 \%, p<0.001)$ in the prevalence of malignant lesions between nodules with and those without echogenic foci. In nodules with echogenic foci, the taller than wide shape $(p=0.026)$, spiculated margin $(p<0.001)$, marked hypoechogenicity $(p<0.001)$ were US features that showed significant difference between benign and malignant nodules. Solid $(n=352,90.3 \%)$ or predominantly solid $(n=38,9.7 \%)$ compositions were the most common features in nodules with echogenic foci (Additional file 1: Table S1). In nodules without echogenic foci, spiculated margin $(p<0.001)$ and marked hypoechogenicity $(p<0.001)$ were US features that showed significant difference. Among malignant lesions with echogenic foci $(n=254), 205$ (80.7\% [205 of 254]) were papillary carcinoma, 6 (2.4\% [6 of 254]) were follicular carcinoma, 32 (12.6\% [32 of 254]) were follicular variant papillary carcinoma, and 11 (4.3\% [11 of 254]) were other histologic types such as medullary carcinoma (Additional file 1: Table S2). Benign lesions varied widely and included follicular adenoma, lymphocytic thyroiditis, and nodular hyperplasia.

The prevalence of benign versus malignant nodules for each 6 types of echogenic foci is summarized in Table 1. All were associated with a relatively high prevalence of malignancy (33.3-77.8\%). Punctate echogenic foci with or without posterior shadowing was the most commonly encountered (53.1\% [207 of 390]) (Fig. 1). Peripheral curvilinear or eggshell echogenic foci with or without shadowing was the least seen (5.4\% [ 21 of 390]). Table 2 demonstrates the malignancy probability of thyroid nodules with suspicious US features according to various malignant risk stratification systems. Among the various TIRADS, the malignancy probability ranged from 70.196.2\%, wherein the ATA guideline exhibited the highest sensitivity.

Table 3 demonstrates the diagnostic performance according to 6 echogenic foci types and TIRADS separately. In regard of echogenic foci type alone, punctate echogenic foci with or without posterior shadowing, punctate echogenic foci with comet-tail artifact, and more than one type of echogenic foci showed the high PPV (73.9, 77.8, and 73.2\% respectively). Macrocalcification and peripheral curvilinear or eggshell echogenic foci showed relatively low PPV, 40.7 and 33.3\% respectively. However, when combined with high suspicion categories of various TIRADS (Table 4), PPV improved in all, especially the improvement was notable in macrocalcification and peripheral curvilinear or eggshell echogenic foci types, to a range of $50.0-80.0 \%$ (Fig. 2). Large echogenic foci without shadowing alone showed PPV of 56.4\%, and when combined with the web-based scoring system, it also improved its PPV to $90.9 \%$. Meanwhile, when we combined 6 types of echogenic foci with non-high suspicion TIRAD category, the PPV decreased to a range of 10.5-57.1\% (Table 5). In comparison of PPV between nodules with echogenic foci and high suspicion or non-high suspicion TIRADS category, all showed statistically significant difference except for punctate echogenic foci with comet-tail artifact combined with K-TIRADS $(85.0 \%$ vs $57.1 \%, p=0.057)$ and ATA guideline $(84.8 \%$ vs $33.3 \% p=0.083)$, macrocalcification combined with the web-based scoring system $(50.0 \%$ vs $40.0 \%, p>0.999)$, K-TIRADS ( $75.0 \%$ vs $34.8 \%, p=0.273$ ), ATA guideline $(75.0 \%$ vs $16.7 \%, p=0.191)$, Russ et al. (66.7\% vs $33.3 \%, p=0.187$ ), and peripheral curvilinear or eggshell echogenic foci combined with web-based scoring system $(66.7 \%$ vs $20.0 \%, p=0.120)$, ATA guideline $(80.0 \%$ vs $16.7 \%, p=0.080)$ and Russ et al. $(66.7 \%$ vs $20.0 \%, p=0.120$ ) (Table 6) (Figs. 3, 4 and 5).

\section{Discussion}

Among the 6 types of echogenic foci we defined, the most common type was punctate echogenic foci with or without posterior acoustic shadowing, referred to as microcalcifications by many authors. This was associated with a high malignancy rate $(73.9 \%)$. Interestingly, punctate echogenic foci with comet-tail artifact were associated with high prevalence of malignancy $(77.8 \%)$. Our result indicates that punctate echogenic foci with comet-tail artifact in solid or predominantly solid nodules should be viewed with suspicion, in accordance with

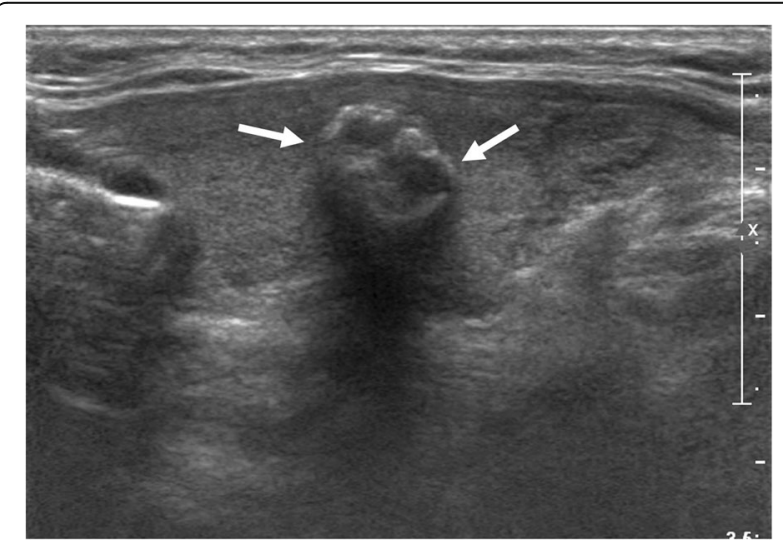

Fig. 2 38-year-old woman with $1.3 \mathrm{~cm}$ solid hypoechoic thyroid nodule. Ultrasound image shows macrocalfication with posterior shadowing (Type 4, arrows). Biopsy finding was papillary carcinoma and confirmed at surgery 
Table 5 Diagnostic Performance of US Characteristics for the Prediction of Malignancy in Thyroid Nodules with Echogenic foci Types with TIRADS Combination (Low suspicion)

\begin{tabular}{|c|c|c|c|c|c|c|c|c|c|}
\hline \multirow{2}{*}{$\begin{array}{l}\text { Echogenic } \\
\text { foci }\end{array}$} & \multirow[t]{2}{*}{ TIRADS } & \multirow[t]{2}{*}{ Total } & \multirow[t]{2}{*}{ Benign } & \multirow[t]{2}{*}{ Malignant } & \multicolumn{5}{|c|}{ Diagnostic Performance } \\
\hline & & & & & Sensitivity & Specificity & Accuracy & PPV & NPV \\
\hline \multirow[t]{4}{*}{ Type 1} & + Web-based TIRADS $(<8)$ & 59 & 40 & 19 & 7.5 & 70.6 & 29.5 & 32.2 & 29.0 \\
\hline & $+\mathrm{K}-$ TIRADS $^{\mathrm{a}}$ & 51 & 35 & 16 & 6.3 & 74.3 & 30.0 & 31.4 & 29.8 \\
\hline & + ATA $^{a}$ & 10 & 8 & 2 & 0.9 & 88.4 & 22.3 & 20.0 & 22.4 \\
\hline & + Russ et al. $(<4 \mathrm{~B})$ & 18 & 13 & 5 & 2.0 & 90.4 & 32.8 & 27.8 & 33.1 \\
\hline \multirow[t]{4}{*}{ Type 2} & + Web-based TIRADS $(<8)$ & 17 & 9 & 8 & 5.2 & 83.3 & 27.1 & 47.1 & 23.7 \\
\hline & $+\mathrm{K}-\mathrm{TIRADS}^{\mathrm{a}}$ & 14 & 6 & 8 & 5.2 & 88.9 & 25.6 & 57.1 & 24.9 \\
\hline & + ATA $^{a}$ & 3 & 2 & 1 & 0.7 & 93.1 & 16.2 & 33.3 & 15.9 \\
\hline & + Russ et al. (<4B) & 7 & 4 & 3 & 2.0 & 92.6 & 25.6 & 42.9 & 25.0 \\
\hline \multirow[t]{4}{*}{ Type 3} & + Web-based TIRADS $(<8)$ & 72 & 39 & 33 & 13.0 & 71.3 & 33.3 & 45.8 & 30.5 \\
\hline & $+\mathrm{K}-$ TIRADS $^{\mathrm{a}}$ & 57 & 35 & 22 & 8.7 & 74.3 & 31.5 & 38.6 & 30.3 \\
\hline & + ATA $^{a}$ & 19 & 17 & 2 & 0.9 & 75.4 & 19.1 & 10.5 & 19.8 \\
\hline & + Russ et al. $(<4 \mathrm{~B})$ & 45 & 28 & 17 & 6.7 & 79.4 & 32.1 & 37.8 & 31.3 \\
\hline \multirow[t]{4}{*}{ Type 4} & + Web-based TIRADS $(<8)$ & 25 & 15 & 10 & 3.9 & 89.0 & 33.6 & 40.0 & 33.2 \\
\hline & $+\mathrm{K}-$ TIRADS $^{\mathrm{a}}$ & 23 & 15 & 8 & 3.1 & 89.0 & 33.1 & 34.8 & 33.0 \\
\hline & + ATA $^{a}$ & 6 & 5 & 1 & 0.5 & 92.8 & 23.0 & 16.7 & 23.2 \\
\hline & + Russ et al. $(<4 \mathrm{~B})$ & 21 & 14 & 7 & 2.8 & 89.7 & 33.1 & 33.3 & 33.1 \\
\hline \multirow[t]{4}{*}{ Type 5} & + Web-based TIRADS $(<8)$ & 15 & 12 & 3 & 1.2 & 91.2 & 32.6 & 20.0 & 33.1 \\
\hline & $+\mathrm{K}$-TIRADS ${ }^{\mathrm{a}}$ & 16 & 13 & 3 & 1.2 & 90.4 & 32.3 & 18.8 & 32.9 \\
\hline & + ATA $^{a}$ & 6 & 5 & 1 & 0.5 & 92.8 & 23.0 & 16.7 & 23.2 \\
\hline & + Russ et al. $(<4 \mathrm{~B})$ & 15 & 12 & 3 & 1.2 & 91.2 & 32.6 & 20.0 & 33.1 \\
\hline \multirow[t]{4}{*}{ Type 6} & + Web-based TIRADS $(<8)$ & 20 & 9 & 11 & 4.3 & 93.4 & 35.4 & 55.0 & 34.3 \\
\hline & $+\mathrm{K}^{-T I R A D S}{ }^{\mathrm{a}}$ & 17 & 10 & 7 & 2.8 & 92.6 & 34.1 & 41.2 & 33.8 \\
\hline & + ATA $^{a}$ & 6 & 4 & 2 & 0.9 & 94.2 & 23.8 & 33.3 & 23.6 \\
\hline & + Russ et al. $(<4 \mathrm{~B})$ & 13 & 8 & 5 & 2.0 & 94.1 & 34.1 & 38.5 & 34.0 \\
\hline
\end{tabular}

TIRADS thyroid image reporting and data system, ATA american thyroid association, PPV positive predictive value, NPV negative predictive value ${ }^{a}$ Other than High suspicion category

Type 1 = Punctate echogenic foci $(\leq 1 \mathrm{~mm})$ with or without posterior shadowing

Type 2 = Punctate echogenic foci with comet-tail artifact

Type 3 = Large echogenic foci ( $>1 \mathrm{~mm}$ ) without shadowing

Type 4 = Macrocalcification (defined as large echogenic foci $(>1 \mathrm{~mm}$ ) with shadowing

Type $5=$ Peripheral curvilinear or eggshell echogenic foci with or without shadowing

Type $6=$ Nodules with more than one type of echogenic foci

the previous study [22]. Moreover, our study demonstrated 3 types of echogenic foci (large echogenic foci without shadowing, macrocalcification, and peripheral curvilinear or eggshell echogenic foci) with relatively low PPV, 33.3-56.4\%. However, when we combined these echogenic foci with various high suspicion categories of TIRADS, the PPV increased. In contrast, when nodules with echogenic foci were combined with non-high suspicion TIRAD category, the PPV decreased. Various TIRADS may further stratify the malignancy risk of nodules with each echogenic foci types. Thus, the combined assessment of TIRADS and echogenic foci is more predictive of malignancy than echogenic foci alone.

Several TIRADS have been developed for malignancy risk stratification $[4,6,27]$ that incorporate US features to categorize thyroid nodules and recommend cytological diagnosis. Korean TIRADS [7] and the ATA guidelines for thyroid nodules stratified the risk of malignancy into several categories based on US patterns [5]. Meanwhile, several attempts have been made to convert this "pattern-based" approach to a "score-based" approach. Representatively, Choi et al. [9] developed a web-based scoring risk stratification system using US characteristics. The TIRADSalso has been recently combined with cytology or scoring systems in management of indeterminate nodules $[28,29]$. Therefore, we hypothesized combining TIRADS with variable echogenic foci would demonstrate promising capacity to distinguish thyroid malignancy with clinical importance. Indeed, the relationship between risk of malignancy and echogenic 
Table 6 Comparison of Positive Predictive Value in Thyroid Nodules with Echogenic foci Types with different TIRADS Category Combination

\begin{tabular}{|c|c|c|c|c|c|}
\hline Echogenic foci & TIRADS & PPV & TIRADS & PPV & $P$ value \\
\hline \multirow[t]{4}{*}{ Type 1} & Web-based TIRADS ( $\geq 8)$ & 90.5 & Web-based TIRADS $(<8)$ & 32.2 & $<0.001$ \\
\hline & K-TIRADS High Suspicion & 87.8 & K-TIRADS ${ }^{a}$ & 31.4 & $<0.001$ \\
\hline & ATA High Suspicion & 87.1 & ATA $^{a}$ & 20.0 & $<0.001$ \\
\hline & Russ et al. ( $\geq 4 \mathrm{~B}$ ) & 78.3 & Russ et al. (<4B) & 27.8 & $<0.001$ \\
\hline \multirow[t]{4}{*}{ Type 2} & Web-based TIRADS ( $\geq 8$ ) & 91.9 & Web-based TIRADS $(<8)$ & 47.1 & $<0.001$ \\
\hline & K-TIRADS High Suspicion & 85.0 & K-TIRADS ${ }^{\mathrm{a}}$ & 57.1 & 0.579 \\
\hline & ATA High Suspicion & 84.8 & ATA $^{a}$ & 33.3 & 0.083 \\
\hline & Russ et al. ( $\geq 4 \mathrm{~B})$ & 83.0 & Russ et al. (<4B) & 42.9 & 0.036 \\
\hline \multirow[t]{4}{*}{ Type 3} & Web-based TIRADS $(\geq 8)$ & 90.9 & Web-based TIRADS $(<8)$ & 45.8 & $<0.001$ \\
\hline & K-TIRADS High Suspicion & 83.8 & K-TIRADS ${ }^{a}$ & 38.6 & $<0.001$ \\
\hline & ATA High Suspicion & 84.2 & ATA $^{a}$ & 10.5 & $<0.001$ \\
\hline & Russ et al. ( $\geq 4 \mathrm{~B})$ & 73.5 & Russ et al. (<4B) & 37.8 & $<0.001$ \\
\hline \multirow[t]{4}{*}{ Type 4} & Web-based TIRADS $(\geq 8)$ & 50.0 & Web-based TIRADS $(<8)$ & 40.0 & $>0.999$ \\
\hline & K-TIRADS High Suspicion & 75.0 & K-TIRADS ${ }^{a}$ & 34.8 & 0.237 \\
\hline & ATA High Suspicion & 75.0 & ATA $^{a}$ & 16.7 & 0.191 \\
\hline & Russ et al. ( $\geq 4 \mathrm{~B})$ & 66.7 & Russ et al. (<4B) & 33.3 & 0.187 \\
\hline \multirow[t]{4}{*}{ Type 5} & Web-based TIRADS ( $\geq 8$ ) & 66.7 & Web-based TIRADS $(<8)$ & 20.0 & 0.120 \\
\hline & K-TIRADS High Suspicion & 80.0 & K-TIRADS ${ }^{\mathrm{a}}$ & 18.8 & 0.025 \\
\hline & ATA High Suspicion & 80.0 & ATA $^{a}$ & 16.7 & 0.080 \\
\hline & Russ et al. ( $\geq 4 \mathrm{~B})$ & 66.7 & Russ et al. (<4B) & 20.0 & 0.120 \\
\hline \multirow[t]{4}{*}{ Type 6} & Web-based TIRADS ( $\geq 8$ ) & 90.5 & Web-based TIRADS $(<8)$ & 55.0 & 0.015 \\
\hline & K-TIRADS High Suspicion & 95.8 & K-TIRADS ${ }^{a}$ & 41.2 & $<0.001$ \\
\hline & ATA High Suspicion & 96.0 & ATA $^{a}$ & 33.3 & $<0.001$ \\
\hline & Russ et al. ( $\geq 4 \mathrm{~B})$ & 89.3 & Russ et al. $(<4 B)$ & 38.5 & $<0.001$ \\
\hline
\end{tabular}

TIRADS thyroid image reporting and data system, ATA american thyroid association, PPV positive predictive value ${ }^{a}$ Other than High suspicion category

TIRADS Thyroid image reporting and data system, ATA American Thyroid Association, PPV Positive Predictive Value

Type 1 = Punctate echogenic foci $(\leq 1 \mathrm{~mm})$ with or without posterior shadowing

Type 2 = Punctate echogenic foci with comet-tail artifact

Type 3 = Large echogenic foci $(>1 \mathrm{~mm}$ ) without shadowing

Type 4 = Macrocalcification (defined as large echogenic foci $(>1 \mathrm{~mm}$ ) with shadowing

Type $5=$ Peripheral curvilinear or eggshell echogenic foci with or without shadowing

Type $6=$ Nodules with more than one type of echogenic foci

foci has been debatable; there have been several attempts to combine several US features with indeterminate echogenic foci of thyroid nodule in predicting the malignancy probability [30, 31]. Frates et al. [30] reported that coarse or rim calcifications double the risk of malignancy as compared with similar nodules without and its risk increases when a nodule is solitary and solid. Kim et al. [31] showed that $82.8 \%$ of malignancy with macrocalcifications are based on triple criteria (marked hypoechogenicity, irregular or microlobulated margin, and a taller than wide shape); the risk of malignancy was $82.8 \%$ in cases with at least 1 of the triple criteria, whereas $34.5 \%$ in cases with no suspicious sonographic finding. Similarly, in our study, by combining various types of echogenic foci with non-high suspicion and high suspicion categories of TIRADS, we observed the changes in diagnostic performance of differentiating benign and malignant thyroid nodules. Combination of TIRADS is more useful for prediction of malignancy risk compared to individual US feature of echogenic foci.

Previous studies have indicated that echogenic foci are more common in malignant thyroid nodules (incidence of $26.0-54.0 \%)$ than in benign lesions (8.0-32.0\%) [15, $16,32]$. This is reflected in our study which revealed significant difference in the prevalence of malignant lesions with and without echogenic foci (nodules with echogenic foci, $65.1 \%$ [254 of 390]; nodules without echogenic foci, $22.6 \%$ [163 of 722]; $p<0.001$ ). Although the presence of echogenic foci is highly suggestive of malignancy $[12,17$, $18,20,21]$, the clinical significance of specific patterns 


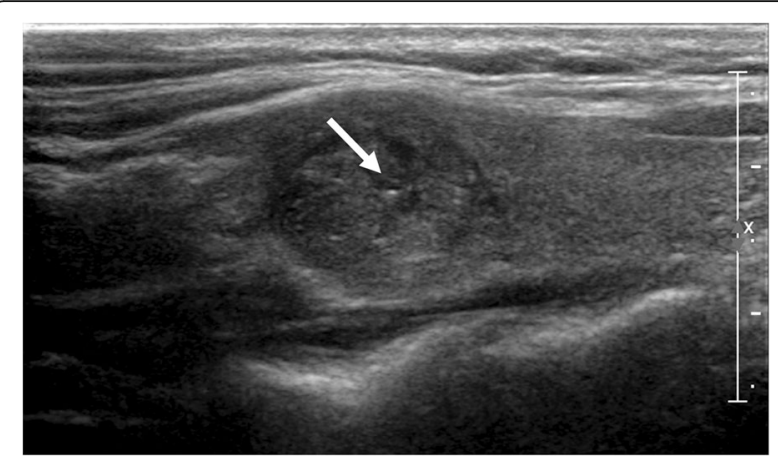

Fig. 3 Echogenic foci associated with malignancy. 36-year-old female with thyroid nodule. Ultrasound image shows a $1.8 \mathrm{~cm}$ hypoechoic, solid nodule. Echogenic foci with no posterior acoustic artifact (Type 1, arrow). Biopsy result was papillary carcinoma and was confirmed at surgery

of echogenic foci is unclear. Echogenic foci range from classic microcalcification associated with papillary thyroid carcinoma to the echogenic foci with comet-tail artifact in benign colloid nodules. Beland et al. [33] found it difficult to categorize $62.0 \%$ of the nodules into either of these echogenic foci; they also found that non-shadowing brightly echogenic linear foci with or without comet-tail artifact is associated with benign nodules. Additionally, the posterior comet-tail artifacts of echogenic foci in cystic nodules have been strongly associated with benignity. Ahuja et al. [34] observed 100 complex cystic nodules with echogenic foci and associated comet-tail artifact were $100 \%$ sensitive and specific for benignity. However, some describe comet-tail artifacts in malignant lesions [33, 35]. Malhi et al. [22] emphasized that comet-tail artifact in hypoechoic nodules should be viewed with suspicion and distinguished from artifacts in cystic components. Likewise, the

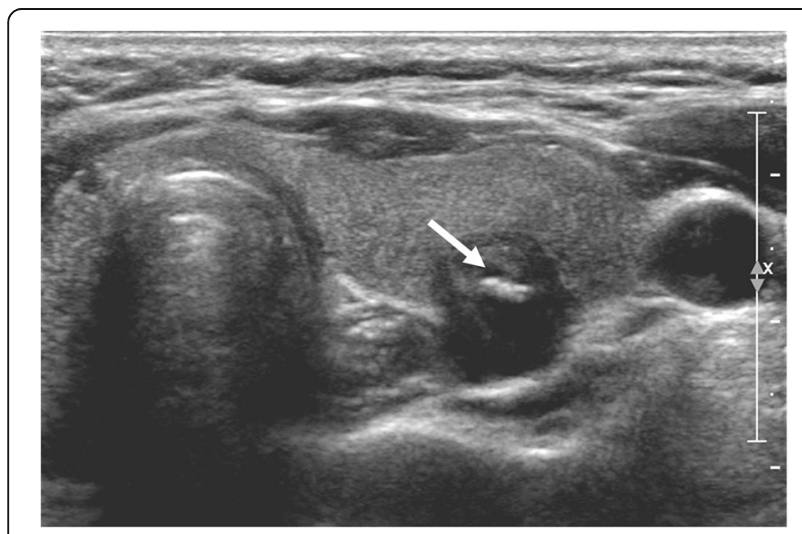

Fig. 4 Echogenic foci associated with malignancy. 51-year-old female with thyroid nodule. Ultrasound image shows a $1.1 \mathrm{~cm}$ hypoechoic, solid nodule with large echogenic foci (> $1 \mathrm{~mm}$ ) without shadowing (Type 3, arrow). Biopsy result was papillary carcinoma and was confirmed at surgery

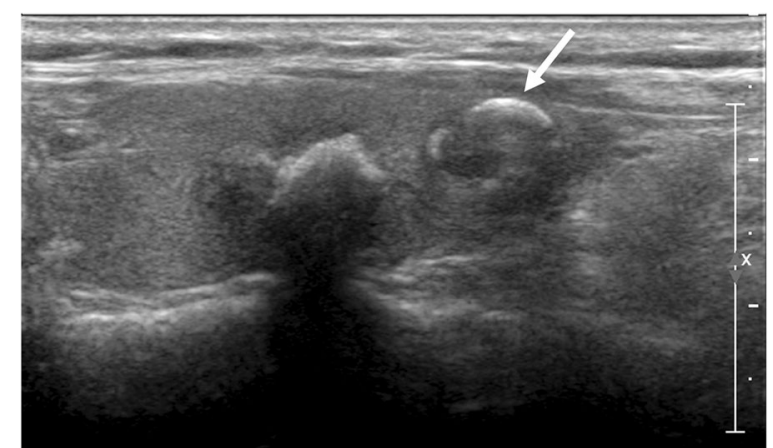

Fig. 5 Echogenic foci associated with malignancy. 70-year-old female with thyroid nodule. Ultrasound image shows a $1.0 \mathrm{~cm}$ hypoechoic, solid nodule with peripheral curvilinear echogenic foci (Type 5, arrow). Biopsy result was papillary carcinoma and was confirmed at surgery

punctate echogenic foci with comet-tail artifact in solid and predominantly solid nodules showed malignancy rate of $77.8 \%$ in our study. The echogenic foci with comet-tail artifacts in the solid portion are not specific for a benign nodule. Indeed, the malignancy risk of punctate echogenic foci of nodule depend on solidity and composition, and comet-tail artifact show relatively high malignancy risk when it is located within hypoechoic solid component. If the proportion of isoechoic or partially cystic nodules with punctate echogenic foci is high or nodules showing echogenic foci within the cystic content is high, the malignancy rate will decrease. Although the exact location of the echogenic foci was not analyzed in this study, the higher predictive value for malignancy by combined TIRADS evaluation may explain the different malignancy risk according to the other US characteristics of nodule. In real practice, we do not recommend for biopsy on the presence of echogenic foci of thyroid nodule alone; rather, we analyze the nodule characteristics such as composition, margin, echogenicity, and size for nodule sampling. We agree that biopsy should be determined after thorough interpretation of thyroid nodule. Nonetheless, our study assessed the association of thyroid malignancy with various echogenic foci and TIRADS in diagnosing thyroid nodule; any type of detected echogenic foci deserves further investigation with suspicion for possible malignancy and consideration of accompanying US features. In addition, although FNA is a good diagnostic tool with a sensitivity of $71.0-93.0 \%$ and a specificity of $96.0 \%$ [11, $36]$, the diagnostic material from calcified nodules is difficult to assess with inadequate results of about $26.8 \%$ [37]. Our study may provide useful information about thyroid nodules with echogenic foci in cases with inadequate cytological results.

The nodules with more than one type of echogenic foci were proven to increase the risk of malignancy from 
$11.2 \%$ for those with a single type to $24.7 \%$ for those having more than one type [22]. Indeed, we encounter many nodules with more than one type of echogenic foci. Several investigations suggest detection of macrocalcifications as well as microcalcifications should increase suspicion of thyroid carcinoma $[11,13,14,30$, 38]. Similarly, in our study, nodules with more than one type of echogenic foci showed a malignancy rate of $73.2 \%$, although not statistically different $(p=0.253)$ due to a small number of cases. However, this result suggests that nodules with more than one type of echogenic foci should be considered suspicious and biopsied.

There are several limitations in our study. First, the appearance of echogenic foci is subjective nature and dependent on factors such as ultrasound machine settings. It is a somewhat dynamic phenomenon that is often more evident on real-time scans. Second, for benign lesions, surgical confirmations were not done and were based on cytopathologic results; this may cause false-negative or false-positive results. Finally, we did not assess the role of isolated echogenic foci or macrocalcification alone as a predictor of malignancy. A previous study demonstrated that thyroid nodules with isolated macrocalcification had a low to intermediate malignancy risk, with a range of 11.4 to $16.1 \%$ [23].

\section{Conclusion}

Overall, the presence of echogenic foci in thyroid nodules is associated with higher rate of malignancy compared to nodules without echogenic foci. Our study showed the usefulness of various TIRADS and its applicability in nodules with six types of echogenic foci. In conclusion, combination with TIRADS could offer better stratification of the malignancy risk of thyroid nodules than individual US feature of echogenic foci and may provide more evidence-based recommendations to patients.

\section{Additional file}

Additional file 1: Table S1. US characteristics of benign and malignancy thyroid nodules with echogenic foci $(n=390)$. Table S2. Histopathologic Results of 417 malignancies. (DOCX 16 kb)

\section{Abbreviations \\ ACR: American College of Radiology; CNB: Core-needle biopsy; FNA: Fine- needle aspiration; KSThR: Korean Society of Thyroid Radiology; NPV: Negative predictive value; PPV: Positive predictive value; TIRADS: Thyroid Imaging Reporting and Data System; US: Ultrasonography}

\section{Acknowledgements}

None applicable

\section{Funding}

This research did not receive any specific grant from funding agencies in the public, commercial, or not-for-profit sectors.

\section{Availability of data and materials}

The datasets used and analyzed during the current study are available from the corresponding author on reasonable request.

\section{Authors' contributions}

J.H.B and S.M.H analyzed and interpreted the patient clinical and imaging data and made consensus. S.M.H was a major contributor in writing and revising the manuscript. J.H.B, H.S.A, S.B.P and Y.J.C acquired and interpreted imaging data of patients, involved in revising the manuscript and approved the final version by ensuring the integrity of the work. J.H.B analyzed the interpretation of overall data more subjectively, revised the contents and approved the final version ensuring possible questions related to the final version work. All authors read and approved the final manuscript.

\section{Ethics approval and consent to participate}

All procedures performed in studies involving human participants were in accordance with the ethical standards of the institutional research committee (Chung-Ang University Hospital) and with the 1964 Helsinki declaration and its later amendments or comparable ethical standards. Our institutional review board (Chung-Ang University Hospital) approved this retrospective study, and the requirement for informed consent was waived.

\section{Consent for publication}

Not applicable

\section{Competing interests}

The authors declare that they have no competing interests.

\section{Publisher's Note}

Springer Nature remains neutral with regard to jurisdictional claims in published maps and institutional affiliations.

\section{Author details}

'Department of Radiology, Chung-Ang University Hospital, Chung-Ang University College of Medicine, 102, Heukseok-ro, Dongjak-gu, Seoul 06973, South Korea. ${ }^{2}$ Department of Internal Medicine, Chung-Ang University Hospital, Chung-Ang University College of Medicine, 102, Heukseok-ro, Dongjak-gu, Seoul 06973, Republic of Korea. ${ }^{3}$ Department of Radiology and Research Institute of Radiology, University of Ulsan College of Medicine, Asan Medical Center, 86 Asanbyeongwon-Gil, Songpa-gu, Seoul 05505, South Korea. ${ }^{4}$ Department of Radiology, Seoul National College of Medicine, 101 Daehak-ro, Jongno-gu, Seoul 110-744, Republic of Korea.

Received: 21 January 2019 Accepted: 26 March 2019

Published online: 04 April 2019

\section{References}

1. Brander A, Viikinkoski P, Nickels J, Kivisaari L. Thyroid gland: US screening in a random adult population. Radiology. 1991;181:683-7.

2. Frates MC, Benson CB, Charboneau JW, Cibas ES, Clark OH, Coleman BG, et al. Management of thyroid nodules detected at US: Society of Radiologists in ultrasound consensus conference statement. Radiology. 2005;237:794800.

3. Tan GH, Gharib H. Thyroid incidentalomas: management approaches to nonpalpable nodules discovered incidentally on thyroid imaging. Ann Intern Med. 1997;126:226-31.

4. Cheng SP, Lee JJ, Lin JL, Chuang SM, Chien MN, Liu CL. Characterization of thyroid nodules using the proposed thyroid imaging reporting and data system (TI-RADS). Head Neck. 2013:35:541-7.

5. Haugen BR, Alexander EK, Bible KC, Doherty GM, Mandel SJ, Nikiforov YE, et al. 2015 American Thyroid Association management guidelines for adult patients with thyroid nodules and differentiated thyroid Cancer: the American Thyroid Association guidelines task force on thyroid nodules and differentiated thyroid Cancer. Thyroid. 2016;26:1-133.

6. Horvath E, Majilis S, Rossi R, Franco C, Niedmann JP, Castro A, et al. An ultrasonogram reporting system for thyroid nodules stratifying cancer risk for clinical management. J Clin Endocrinol Metab. 2009;94:1748-51.

7. Shin JH, Baek JH, Chung J, Ha EJ, Kim JH, Lee YH, et al. Ultrasonography diagnosis and imaging-based Management of Thyroid Nodules: revised Korean Society of Thyroid Radiology Consensus Statement and Recommendations. Korean J Radiol. 2016;17:370-95. 
8. Ha EJ, Moon WJ, Na DG, Lee YH, Choi N, Kim SJ, et al. A multicenter prospective validation study for the Korean thyroid imaging reporting and data system in patients with thyroid nodules. Korean J Radiol. 2016:17:811-21.

9. Choi YJ, Baek JH, Baek SH, Shim WH, Lee KD, Lee HS, et al. Web-based malignancy risk estimation for thyroid nodules using ultrasonography characteristics: development and validation of a predictive model. Thyroid. 2015:25:1306-12.

10. Russ G, Royer B, Bigorgne C, Rouxel A, Bienvenu-Perrard M, Leenhardt $L$. Prospective evaluation of thyroid imaging reporting and data system on 4550 nodules with and without elastography. Eur J Endocrinol. 2013; 168:649-55.

11. Seiberling KA, Dutra JC, Grant T, Bajramovic S. Role of intrathyroidal calcifications detected on ultrasound as a marker of malignancy. Laryngoscope. 2004;114:1753-7.

12. Takashima S, Fukuda H, Nomura N, Kishimoto H, Kim T, Kobayashi T. Thyroid nodules: re-evaluation with ultrasound. J Clin Ultrasound. 1995:23:179-84.

13. Taki S, Terahata S, Yamashita R, Kinuya K, Nobata K, Kakuda K, et al. Thyroid calcifications. sonographic patterns and incidence of cancer Clin Imaging. 2004;28:368-71.

14. Wang N, Xu Y, Ge C, Guo R, Guo K. Association of sonographically detected calcification with thyroid carcinoma. Head Neck. 2006;28:1077-83.

15. Kakkos SK, Scopa CD, Chalmoukis AK, Karachalios DA, Spiliotis JD, Harkoftakis $J G$, et al. Relative risk of cancer in sonographically detected thyroid nodules with calcifications. J Clin Ultrasound. 2000;28:347-52.

16. Khoo ML, Asa SL, Witterick IJ, Freeman JL. Thyroid calcification and its association with thyroid carcinoma. Head Neck. 2002;24:651-5.

17. Fukatsu H, Makino N, Kodama Y, Ikeda M, Ishigaki T, Sakuma S. Evaluation of thyroid calcification using computed radiography with image plate. Eur J Radiol. 1989;9:22-8.

18. Bruneton JN, Balu-Maestro C, Marcy PY, Melia P, Mourou MY. Very high frequency $(13 \mathrm{MHz})$ ultrasonographic examination of the normal neck: detection of normal lymph nodes and thyroid nodules. J Ultrasound Med. 1994;13:87-90

19. Peccin S, de Castsro JA, Furlanetto TW, Furtado AP, Brasil BA, Czepielewski MA. Ultrasonography: is it useful in the diagnosis of cancer in thyroid nodules? J Endocrinol Investig. 2002;25:39-43.

20. Tessler FN, Middleton WD, Grant EG, Hoang JK, Berland LL, Teefey SA, et al. ACR thyroid imaging, reporting and data system (TI-RADS): white paper of the ACR TI-RADS Committee. J Am Coll Radiol. 2017;14:587-95.

21. Watters DA, Ahuja AT, Evans RM, Chick W, King WW, Metreweli C, et al. Role of ultrasound in the management of thyroid nodules. Am J Surg. 1992;164:654-7.

22. Malhi H, Beland MD, Cen SY, Allgood E, Daley K, Martin SE, et al. Echogenic foci in thyroid nodules: significance of posterior acoustic artifacts. AJR Am J Roentgenol. 2014;203:1310-6.

23. Na DG, Kim DS, Kim SJ, Ryoo JW, Jung SL. Thyroid nodules with isolated macrocalcification: malignancy risk and diagnostic efficacy of fine-needle aspiration and core needle biopsy. Ultrasonography. 2016;35:212-9.

24. Cibas ES, Ali SZ, NCITFSotS C. The Bethesda system for reporting thyroid cytopathology. Am J Clin Pathol. 2009;132:658-65.

25. Jung CK, Min HS, Park HJ, Song DE, Kim JH, Park SY, et al. Pathology reporting of thyroid Core needle biopsy: a proposal of the Korean endocrine pathology thyroid Core needle biopsy study group. J Pathol Transl Med. 2015:49:288-99.

26. Russ G. Risk stratification of thyroid nodules on ultrasonography with the French TI-RADS: description and reflections. Ultrasonography. 2016;35:25-38.

27. Kwak JY, Han KH, Yoon JH, Moon HJ, Son EJ, Park SH, et al. Thyroid imaging reporting and data system for US features of nodules: a step in establishing better stratification of cancer risk. Radiology. 2011;260:892-9.

28. Ulisse S, Bosco D, Nardi F, Nesca A, D'Armiento E, Guglielmino V, et al. Thyroid imaging reporting and data system score combined with the new Italian classification for thyroid cytology improves the clinical Management of Indeterminate Nodules. Int J Endocrinol. 2017;2017:9692304.

29. He YP, Xu HX, Zhao CK, Sun LP, Li XL, Yue WW, et al. Cytologically indeterminate thyroid nodules: increased diagnostic performance with combination of US TI-RADS and a new scoring system. Sci Rep. 2017;7:6906.

30. Frates MC, Benson CB, Doubilet PM, Kunreuther E, Contreras M, Cibas $E S$, et al. Prevalence and distribution of carcinoma in patients with solitary and multiple thyroid nodules on sonography. J Clin Endocrinol Metab. 2006;91:3411-7.
31. Kim MJ, Kim EK, Kwak JY, Park CS, Chung WY, Nam KH, et al. Differentiation of thyroid nodules with macrocalcifications: role of suspicious sonographic findings. J Ultrasound Med. 2008;27:1179-84.

32. Koike E, Noguchi S, Yamashita H, Murakami T, Ohshima A, Kawamoto H, et al. Ultrasonographic characteristics of thyroid nodules: prediction of malignancy. Arch Surg. 2001;136:334-7.

33. Beland MD, Kwon L, Delellis RA, Cronan JJ, Grant EG. Nonshadowing echogenic foci in thyroid nodules: are certain appearances enough to avoid thyroid biopsy? J Ultrasound Med. 2011;30:753-60.

34. Ahuja A, Chick W, King W, Metreweli C. Clinical significance of the comettail artifact in thyroid ultrasound. J Clin Ultrasound. 1996;24:129-33.

35. Bonavita JA, Mayo J, Babb J, Bennett G, Oweity T, Macari M, et al. Pattern recognition of benign nodules at ultrasound of the thyroid: which nodules can be left alone? AJR Am J Roentgenol. 2009;193:207-13.

36. Amrikachi M, Ramzy I, Rubenfeld S, Wheeler TM. Accuracy of fine-needle aspiration of thyroid. Arch Pathol Lab Med. 2001;125:484-8.

37. Park M, Shin JH, Han BK, Ko EY, Hwang HS, Kang SS, et al. Sonography of thyroid nodules with peripheral calcifications. J Clin Ultrasound. 2009;37: 324-8.

38. Consorti F, Anello A, Benvenuti C, Boncompagni A, Giovannone G, Moles N, et al. Clinical value of calcifications in thyroid carcinoma and multinodular goiter. Anticancer Res. 2003;23:3089-92.
Ready to submit your research? Choose BMC and benefit from:

- fast, convenient online submission

- thorough peer review by experienced researchers in your field

- rapid publication on acceptance

- support for research data, including large and complex data types

- gold Open Access which fosters wider collaboration and increased citations

- maximum visibility for your research: over $100 \mathrm{M}$ website views per year

At BMC, research is always in progress.

Learn more biomedcentral.com/submissions 